\title{
ASPECTOS BÁSICOS DA LESÃO DE ISQUEMIA E REPERFUSÃO E DO PRÉ-CONDICIONAMENTO ISQUÊMICO ${ }^{1}$
}

\author{
BASICS ASPECTS OF THE ISCHEMIA REPERFUSION \\ INJURY AND OF THE ISCHEMIC PRECONDITIONING ${ }^{1}$
}

\author{
Orlando de Castro e Silva Jr. ${ }^{2}$ \\ Sergio Centurion ${ }^{3}$ \\ Eduardo G. Pacheco ${ }^{3}$ \\ João Luiz Brisotti ${ }^{3}$ \\ Alexandre Ferreira Oliveira ${ }^{3}$ \\ Karina Dal Sasso ${ }^{4}$
}

\section{RESUMO}

A isquemia tem papel fundamental em muitas situações clínicas perioperatórias . Apesar da revascularização sanguínea a um órgão isquêmico seja essencial para prevenir a irreversibilidade da lesão celular, a reperfusão pode agravar as lesões produzidas na fase isquêmica isolada. Assim, o dano celular induzido após reperfusão de um órgão isquêmico é denominado de lesão de isquemia-reperfusão (I/R). Aspectos básicos da lesão I/R, são revisados neste artigo. Disponível em URL: http://www.scielo.br/acb.

Descritores - Isquemia, reperfusão, isquemia/ reperfusão, fígado.

\begin{abstract}
Ischemia contributes to the pathogenesis of many perioperative clinical conditions. Although restoration of blood flow to an ischemic organ is essencial to prevent irreversible cellular injury, reperfusion may augment tissue injury in excess to that produced by ischemia alone. Thus, cellular damage after reperfusion of previously viable ischemic tissue is defined as ischemia-reperfusion injury. The basic aspects of $I / R$ are reviewed.
\end{abstract}

Key Words - ischemia, reperfusion, ischemiareperfusion, liver.

\section{INTRODUÇÃO}

A isquemia contribui para a fisiopatologia de condições vivenciadas por cirurgiões e anestesistas, como por exemplo infarto do miocárdio, insuficiência vascular periférica e choque hipovolêmico. Apesar da restauração do fluxo sanguíneo a um órgão isquêmico seja essencial para prevenir dano celular irreversível, a reperfusão per se pode agravar o dano celular isquêmico.Por exemplo, as alterações histológicas induzidas por 3 horas de isquemia intestinal, seguidas por uma hora de reperfusão, são mais graves do que as induzidas por 4 horas de isquemia intestinal isolada.

Períodos de isquemia induzidos por exclusão vascular do fígado por clampagem da tríade portal ou total do fígado com clampagem concomitante da cava supra - hepática ${ }^{1}$ são muitas vezes necessários durante ressecções hepáticas parciais, pelo risco potencial de sangramento, de intensidade variáveis, sobretudo no momento da ressecção do parênquima hepático. Sabese que a isquemia, ao mesmo tempo que evita ou minimiza sangramentos durante a cirurgia, induz diferentes intensidades de sofrimento (isquemia/ reperfusão) (I/R) hepatocelular. Dentro de determinados limites, pacientes suportam melhor a isquemia hepática do que as consequências de grandes hemorragias e consequentes transfusões de sangue e derivados ${ }^{2,3}$.

\section{ASPECTOS BÁSICOS DA LESÃO DE ISQUEMIA-REPERFUSÀO}

Sabe-se que as conseqüências da isquemia, em diferentes tecidos, depende de sua duração, e que muitas das lesões são desenvolvidas durante o estágio de

\footnotetext{
1 Grupo Integrado de Transplante de Fígado. Projeto Temático FAPESP

2 Professor Associado do Departamento de Cirurgia e Anatomia da FMRP-USP

Pós-Graduandos do Departamento de Cirurgia e Anatomia da FMRP-USP

Enfermeira do Grupo Integrado de Transplante de Fígado.
} 
reoxigenação decorrente da reperfusão tecidual ${ }^{4,5,6}(\mathrm{Fi}$ gura 1). As mitocôndrias são alvos importantes dos danos provocados pelos processos de isquemia e reperfusão ${ }^{7}$ Nelas ocorrem diminuição das atividades da nicotinamida adenina dinucleotídeo ligada com hidrogênio (NADH) desidrogenase, do carreador de adenosina difosfato / adenosina trifosfato (ADP/ATP) e da ATP sintetase, além do aumento na atividade da fosfolipase A2. Ocorre ainda, acentuado acúmulo de cálcio e aumento da geração de radicais livres pelas mitocôndrias. A associação destes eventos pode ser responsável pelas lesões e morte celular, decorrentes da reperfusão, possivelmente por um fenômeno de transição de permeabilidade da membrana mitocondrial interna ${ }^{8}$

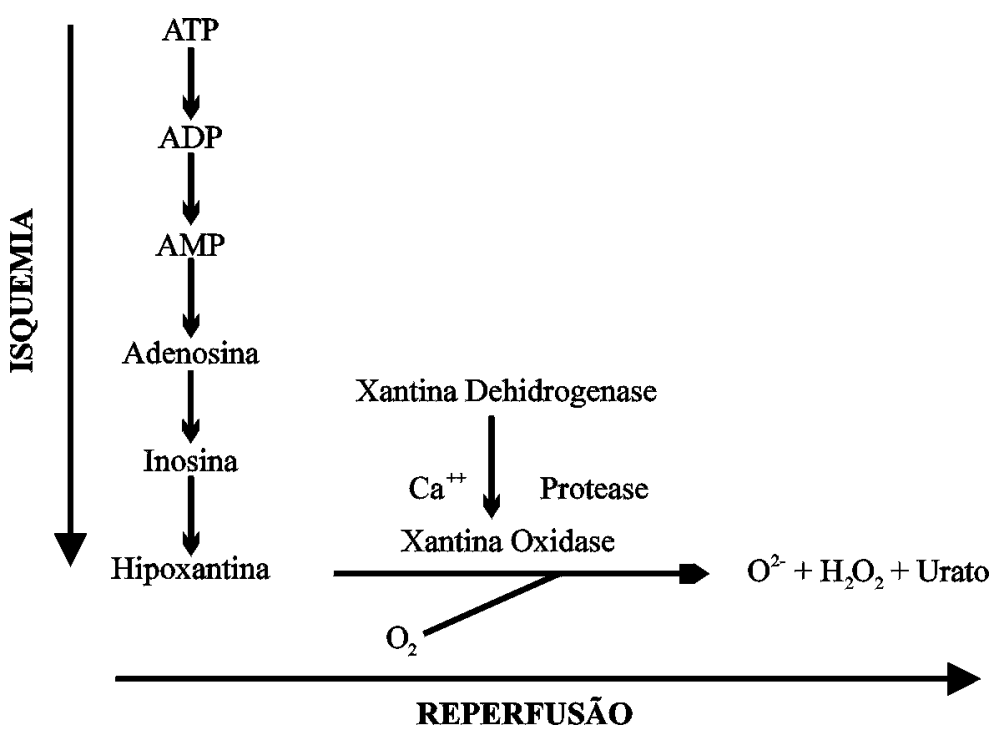

Figura 1 - Mecanismo proposto para geração de radicais livres de oxigênio após períodos de isquemia e reperfusão ${ }^{4,5,6}$.

No sentido de se proteger o fígado das lesões de isquemia/reperfusão ( $\mathrm{I} / \mathrm{R})$ vários tipos de proteção hepática têm sido utilizados, como uso de drogas ${ }^{9,10}$, hipotermia $^{11,12}$, pré-condicionamento isquêmico e indução de isquemia/reperfusão sucessivas por clampagens intermitentes do pedículo hepático por tempos diferentes ${ }^{1,13}$.

Vários estudos experimentais têm demonstrado a eficácia de drogas no sentido de prevenir ou atenuar as lesões de isquemia e reperfusão. Têm sido testadas a superóxido dismutase, catalase, manitol, alopurinol, vitamina $\mathrm{E}, \mathrm{N}$-acetilcisteína, compostos quelantes de ferro, inibidores da enzima conversora da angiotensina e antagonistas dos canais de cálcio ${ }^{1,14}$.

Em estudos anteriores observou-se que grupos de ratos com período de isquemia de 25 minutos, com 5 minutos de reperfusão em tempos sucessivos até completar 90 minutos apresentaram valores da respiração mitocondrial significativamente menores do que o grupo onde o tempo de isquemia foi de 15 minutos, seguido de 15 minutos de reperfusão em 3 tempos sucessivos, completando 90 minutos $^{13}$. Nesse mesmo estudo, o período menor de isquemia, 15 minutos, pode ter atuado como pré-condicionamento isquêmico do fígado como também foi descrito por outros autores ${ }^{14,15}$. A desvantagem técnica da clampagem intermitente, nas hepatectomias parciais é a possibilidade de sangramento que ocorre durante os momentos de desclampagens para reperfusão do fígado.

O termo pré-condicionamemto isquêmico foi introduzido na literatura por Murry et al., $1986^{16}$ e significa indução de um pequeno período de isquemia seguido por pequeno período de reperfusão antes de um período mais longo de isquemia. Eles descreveram o efeito benéfico de curtos episódios de oclusão coronária seguidos também de curtos períodos de reperfusão no tamanho da área de infarto causado por isquemia sem pré-condicionamento. Resultados semelhantes foram descritos por outros autores ${ }^{17}$. O papel do précondicionamento isquêmico no aumento da tolerância à isquemia tem sido descrito em vários órgãos como coração, cérebro, medula espinhal, músculo esquelético, retina, rins, intestino, além de fígado. Entretanto, apesar de estudos sobre o porque do efeito protetor do pré-condicionamento isquêmico e seu real fundamento não esta ainda claramente estabelecido ${ }^{17}$. 
Em 1908, Pringle ${ }^{1}$ descreveu a técnica de pinçamento da tríade portal para indução de isquemia hepática para coibir sangramentos hepáticos, e que desde então, tem sido muito utilizada nas cirurgias por trauma hepático e nas ressecções hepáticas segmentares ou amplas.

Nos organismos submetidos a isquemia hepática, seguida de reperfusão, ocorre interação complexa entre alterações microvasculares, liberação de mediadores inflamatórios, radicais livres de oxigênio, ativação de neutrófilos, plaquetas, células de Kupffer e células endoteliais sinusoidais. A ativação destas células pode levar à liberação de fator de necrose tumoral (TNF), leucotrienos, tromboxanos, prostaglandinas, endotelinas fator de ativação plaquetária, causando danos à membrana celular, membrana mitocondrial interna e endotélio levando a distúrbios na microcirculação hepática ${ }^{14}$ (Figura 2).

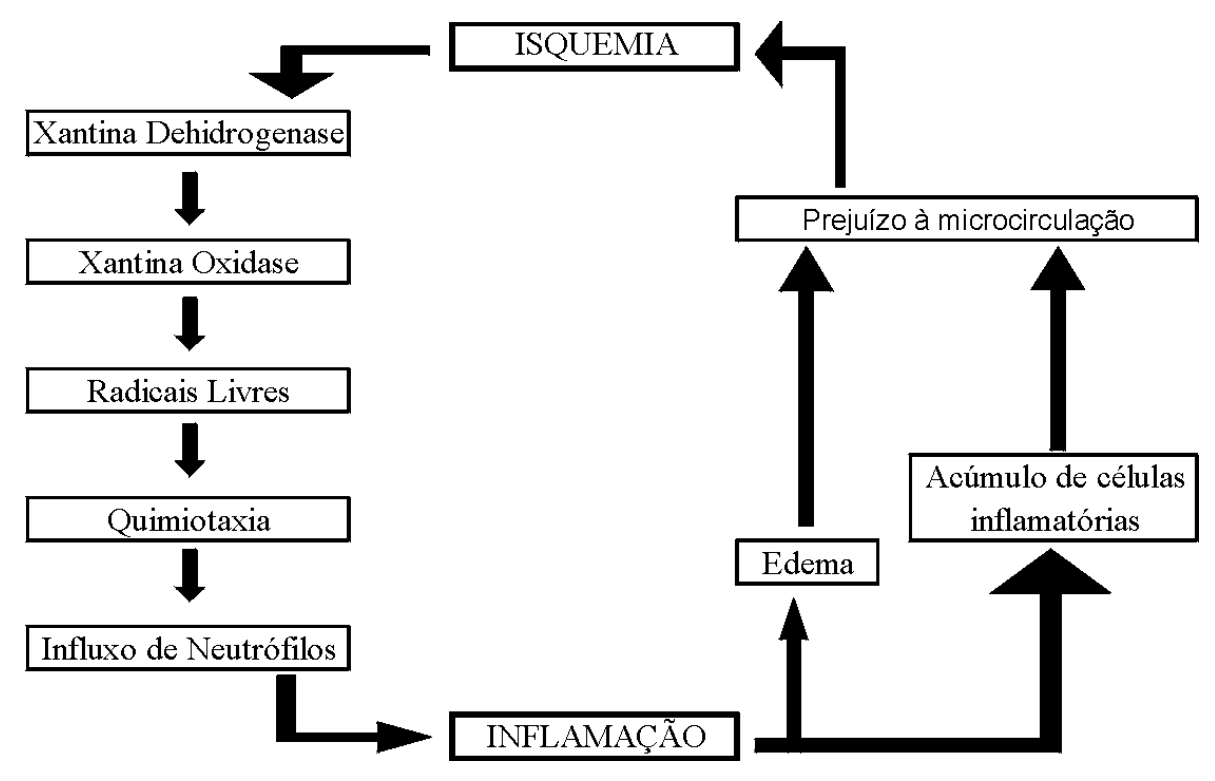

Figura 2 - Seqüência de eventos relacionando a lesão de isquemia e reperfusão e a lesão inflamatória.

Dependendo do tempo de isquemia e do momento da reperfusão, podem haver desde simples alterações bioquímicas até necrose celular. O pré-condicionamento isquêmico tem minimizado essas alterações, funcionando como possível método protetor das lesões hepatocelulares induzidas pelo binômio isquemia/ reperfusão ${ }^{14,17}$ (Tabela 1).

Tabela 1 - Alterações celulares induzidas pela isquemia- reperfusão. ${ }^{14}$

1. Alteração do potencial de membrana

2. Alteração da distribuição de íons - aumento da relação $\mathrm{Ca}^{++} / \mathrm{Na}^{+}$intracelular

3. Edema celular

4. Desorganização do citoesqueleto de órgãos e tecidos

5. Diminuição da fosfocreatina

6. Acidose celular

Com 40 minutos de isquemia, não se tem verificado nos grupos isquêmicos com e sem pré - condicionamento, alterações dos níveis das bilirrubinas totais e frações. Esses indicadores são poucos sensíveis, tanto da isquemia como de necrose hepatocelular, sobretudo no tempo de isquemia estudado. Nas hepatectomias parciais, observa-se aumento das bilirrubinas direta e indireta na vigência de isquemia hepática em função de agressão adicional ao fígado remanescente após a ressecção hepática ${ }^{10}$. 
Com relação às aminotransferases, em tempos variados de isquemia, tem-se verificado aumento significativo da ALT e da AST em grupos isquêmicos, em relação à controles. Boin, $1997^{10}$ não verificou aumento destas enzimas após tempos de isquemia entre 60 e 120 minutos, mas sim após reperfusão do fígado. Estudos de I/R, realizados em nosso laboratório, por sua vez, os níveis séricos de ALT e AST, no grupo com pré - condicionamento, foram semelhantes ao grupo isquêmico sem pré - condicionamento ${ }^{18}$. Isto mostra que, do ponto de vista enzimático do sangue, o pré-condicionamento isquêmico não foi capaz de minimizar as lesões induzidas pela isquemia. As aminotransferases são, atualmente, os marcadores mais sensíveis da lesão iquêmica do fígado ${ }^{17,19}$. Clavien et $a l^{19}$ utilizaram essas enzimas, no primeiro estudo com pré-condicionamento isquêmico, realizado em seres humanos, para mostrar o efeito benéfico do pré-condicionamento isquêmico em hepatectomias parciais com exclusão vascular. Eles verificaram diminuição significativa da ALT e AST no grupo de pacientes submetidos a hepatectomia parcial, isquemia e précondicionamento isquêmico. No mesmo estudo com isquemia e pré condicionamento isquêmico, o comportamento sérico da DHL foi semelhante ao das aminotransferases; houve aumento significativo no grupo isquêmico com redução no grupo onde se usou o pré condicionamento isquêmico do fígado ${ }^{19}$.

A ativação das células de Kupffer e neutrófilos induzida pela isquemia e reperfusão pode ser intemediada pelo fator de ativação plaquetária ${ }^{10}$, propiciando a liberação de radicais livres de oxigênio, aumento do influxo de cálcio ${ }^{1}$ e redução de agentes antioxidantes superóxido dismutase, catalase e glutationa, aumentando a produção de malondialdeído (MDA). A ativação de fosfolipases pode produzir graves danos aos lípides de membrana e alterações da permeabilidade mitocondrial ${ }^{7}$.

Usualmente, quando o tecido hepático é submetido a isquemia, ocorre diminuição da velocidade de consumo de oxigênio no estado 3 da respiração mitocondrial e aumento do consumo no estado 4 . O estado 3 representa a capacidade de fosforilação mitocondrial e consequente produção de energia (fosforilação do ADP em ATP). O estado 4 significa respiração no estado basal, responsável pela geração e manutenção do potencial de membrana mitocondrial e está relacionado com a integridade da membrana mitocondrial. A Razão de controle respiratório é a razão entre estado 3 e $4^{20}$.

No fígado isquêmico há diminuição significativa do estado 3 mostrando diminuição da produção de energia mitocondrial, induzida pela isquemia ${ }^{10,21}$. Os níves do estado 3 de grupos isquêmicos com précondicionamento são semelhantes a controles, sugerindo que o pré-condicionamento protege as mitocôndrias da lesão isquêmica. Com relação ao estado 4 não há alteração de seus valores em grupos isquêmicos estudados, mostrando que, em períodos inferiores a uma hora de isquemia normotérmica, o transporte de elétrons pela cadeia respiratória da membrana mitocondrial mantém-se inalterado. Nesses estudos o potencial de membrana, nos grupos isquêmicos também permanece semelhante aos controles, o que confirma os resultados obtidos em relação ao estado 4, ou seja, que o transporte de elétrons é eficiente na formação e manutenção do potencial de membrana mitocondrial, tanto com, como sem pré-condicionamento.

Uma das bases do mecanismo quimiosmótico de transdução de energia é a impermeabilidade da membrana mitocondrial interna a solutos que não sejam dotados de sistemas específicos de transporte. A impermeabilidade de membrana pode ser afetada por ação de radicais livres na membrana mitocondrial ou em decorrência da transição de permeabilidade da mesma ${ }^{22}$. A transição de permeabilidade mitocondrial é um fenômeno caracterizado por aumento não seletivo na permeabilidade da membrana mitocondrial interna, mediada por $\mathrm{Ca}^{+2}$ mais inúmeros agentes indutores, dentre eles, espécies reativas do oxigênio (radicais livres). A presença de $\mathrm{Ca}^{+2}$ na matriz mitocondrial é o requisito mais importante para a indução da permeabilidade da membrana ${ }^{22}$.

Tem-se observado, que sob determinadas condições, ocorre aumento de permeabilidade das mitocôndrias seguido de intumescimento osmótico da matriz (swelling) e muitas evidências apontam para a participação de espécies reativas do oxigênio no processo ${ }^{23}$. Em presença de $\mathrm{Ca}^{+2}$, o grupo que sofre isquemia tem intumescimento osmótico mitocondrial (swelling) mais acentuado, tanto em relação a controles como ao grupos com pré-condicionamento isquêmico, sugerindo possível participação de espécies reativas do oxigênio na indução do processo, que por sua vez seria prevenido pelo pré-condicionamento isquêmico diminuindo a formação dessas espécies, por mecanismos protetores mitocondriais. Além disso, o aumento do intumescimento osmótico mitocondrial na isquemia poderia ser justificado pelo desequilíbrio na homeostase mitocondrial de $\mathrm{Ca}^{+2}$ causado pelo tempo de isquemia, o qual foi submetido esses animais.

Em suma, o pré-condicionamento isquêmico diminui os níveis de malondialdeído (MDA) em relação a animais com isquemia. Assim, é possível concluir que a proteção do pré-condicionamento isquêmico verificado em estudos anteriores deve-se, entre outros fatores, a inibição de espécies reativas do oxigênio e conseqüente diminuição da lipoperoxidação. 


\section{REFERÊNCIAS}

1. Cerwenka H, Mischinger. Postischemic oxidative stress during liver resection. In Book proceedings of $3 \mathrm{rd}$ word congress of the IHPBA. Ed. Gonzales, E.M.; Paschoal, M.H., Madrid, 1999. p.160-4.

2. Makuuchi M, Takayama T, Gunven P. Restrictive versus liberal blood transfusion policy for hepatectomies in cirrothic patients. Word J Surg 1989;13:644-8.

3. Belghiti J, Noun R, Zante E. Portal triade clamping or hepatic vascular exclusion for major liver resection; a controlled study. Ann Surg 1996;224:155-61.

4. Aalto TK, Raivio KO. Nuclotide depletion due to reative oxygen metabolites in endothelial cells: effects of antioxidants. Pediatric Res 1993;34:572-76.

5. Zhang J, Dawson VL, Dalson TM. Nitric oxide activation of poly (ADP-ribose) synthetase in neurotoxicity. Science 1994;263:687-9.

6. Szabo C, Zingarelli B, Salzman AL. Role of poly- ADP ribosyltransferase activation in the vascular contractile and energetic failure elicited by exougenous and endogenous nitric oxide and peroxinitrite. Circ Res 1996;78:1051-63.

7. Farber JL, Chien KR, Mittnnacht S. Jr. The pathogenesis of irreversible cell injury in ischemia. Am J Pathol 1981; 102:271-81

8. KonoY, Osawa K, Tanaka J. Significance of mitochondrial enhancement inrestoring hepatic energy charge after revascularization of isolated ischemic liver. Transplatation 1982;33:150-5

9. Brisotti JL, Picinato MANC, Franco CFF. Efeito da deferoxamina na isquemia e reperfusão do fígado remanescente após ressecção hepática parcial. Acta Cir Bras 2000; 14:39-42.

10. Boin IFSF. Atuação do WEB 2086 como inibidor da ação do fator de ativação plaquetária (PAF) em ratos wistar submetidos à isquemia hepática seltiva normotérmica e reperfusão. Tese de doutorado UNICAMP, 1997, 110 pp.

11. Hannoun L, Delriviere L, Gibbs P. Major extended liver resection in dislased livers using hypothermic protection: preminary results from the first 12 patients treated with this new technique. J Am Coll Surg 1996;183:597-605.

12. Vaillant JC, Borie DC, Hannoun L. Hepatectomy with hypothermic perfusion of the liver. Hepato Gastroenterol 1998;445:381-8.

13. Alves Neto WC, Jordani MC, Souza MEJ, Franco CFF, Picinato MANC, Castro e Silva Jr. O. Repercusões teciduais do fígado em dois modelos de isquemia e reperfusão intermitentes. Acta Cir. Bras 1998;13 (supl.1):38-9.

14. Collard CD, Gelman S. Patophysiology, Clinical manifestations and prevention of ischemia - reperfusion injury. Anesthesiology 2001;94:1-11.

15. Belghiti J, Noun R, Malafosse R. Continous versus intermittent portal triad clamping for liver resection. A controlled study. Ann Surg 1999;229:369-75.

16. Murry CE, Jennings RB, Reimer KA. Preconditioning with ischaemia: a delay of lethal cell injury in ischemic myocardium. Circultion 1986;74:1124-36.

17. Bulkley GB. Preconditioning for protection from ischemia injury: Discriminating cause from the effect from epiphenomenon. Ann Surg 2000;232:163-5.

18. Centurion SAR. Avaliação da função mitocondrial do fígado submetido a isquemia parcial com pré-condicionamento e sem pré-condicionamento isquêmico. Tese de Doutorado FMRPUSP. 2002, in press.

19. Clavien PA, Yadav S, Sindram D. Protective effects of ischemic preconditioning for liver resection performed under inflow occlusion in humans. Ann Surg 2000;232:155-62.

20. Curti C, Uyemura SA. Estudo da fosforilação oxidativa. In: Modelos experimentais de pesquisa em cirurgia. Ed. Castro e Silva Jr., O.; Zucoloto, S.; Beer Jr., A. Robe Editorial. São Paulo, 1998; p.149-58.

21. Iu S, Harvey PRC, Makowka L. Markers of allografth viability in the rat. Relations hip betwen transplantation viability and liver function in the isolated perfused rat liver. Transplantation 1987;45:562-9.

22. Zoratti M, Szabo I. The mitochondrial permeabilty transition. Biochim. Biophys Acta 1995;1241:139-76.

23. Pedersen PL, Greenawalt JW, Reynafarje B, Hullihen J, Deccker GL, Soper J W, Bustamente E. Preparation and characterization of mitochondria and submitochondrial particles of rat liver and liver-derived tissues. Meth Cell Biol 1978;20: 411-81.

\section{Endereço para Correspondência}

Orlando de Castro e Silva Jr

Departamento de Cirurgia e Anatomia

Faculdade de Medicina de Ribeirão Preto - USP

Campus Universitário - Monte Alegre

14049-990 - Ribeirão Preto - SP

e-mail-orlandocjs@hotmail.com

Conflito de interesse: nenhum

Fonte de Financiamento: FAPESP 